\title{
The Effects of Concurrent Resistance And Endurance Training Follow a Detraining Period in Elementary School StUdents
}

\author{
Albano P. Santos, ${ }^{1,2}$ Daniel A. Marinho, ${ }^{1,2}$ Aldo M. Costa, ${ }^{1,2}$ Mikel Izquierdo, ${ }^{3}$ \\ and Mário C. Marques ${ }^{1,2}$ \\ ${ }^{1}$ Department of Sport Sciences, University of Beira Interior, Covilhã, Portugal; ${ }^{2}$ Sport Sciences, Exercise and Health Department, \\ University of Trás- os-Montes e Alto Douro; Vila Real de Trás-os-Montes, Portugal; and ${ }^{3}$ Department of Health Sciences, Public \\ University of Navarre, Navarre, Spain
}

\begin{abstract}
Santos, AP, Marinho, DA, Costa, AM, Izquierdo, M, and Marques, MC. The effects of concurrent resistance and endurance training follow a detraining period in elementary school students. J Strength Cond Res 26(6): 1708-1716, 2012-The purpose of this study was to compare the effects of an 8-week training period of resistance training alone (GR), or combined resistance and endurance training (GCOM), followed by 12 weeks of detraining (DT) on body composition, explosive strength, and $\dot{\mathrm{V}}_{2}$ max adaptations in a large sample of adolescent school boys. Forty-two healthy boys recruited from a Portuguese public high school (age: $13.3 \pm 1.04$ years) were assigned to 2 experimental groups to train twice a week for 8 weeks: GR ( $n=15), \mathrm{GCOM}(n=15)$, and a control group (GC: $n=12$; no training program). Significant training-induced differences were observed in 1- and 3-kg medicine ball throw gains (GR: +10.3 and $+9.8 \%$, respectively; GCOM: +14.4 and $+7 \%$, respectively), whereas no significant changes were observed after a DT period in both the experimental groups. Significant training-induced gains in the height and length of the countermovement (vertical-and-horizontal) jumps were observed in both the experimental groups. No differences were perceived after a DT period in lower limb power. Time at $20 \mathrm{~m}$ decreased significantly for both intervention programs (GR: $-11.5 \%$ and GCOM: $-12,4 \%,<0.00$ ), but either GR or GCOM groups kept the running speed after a DT period of 12 weeks. After training, the $\dot{\mathrm{V}}_{2}$ max increased only significantly for GCOM (4.6\%, $p=0.01)$. A significant loss was observed after a DT period in GR but not in GCOM. Performing resistance and endurance training in the same workout does
\end{abstract}

Address correspondence to Dr. Mário C. Marques, mariomarques@ mariomarques.com.

26(6)/1708-1716

Journal of Strength and Conditioning Research

(C) 2012 National Strength and Conditioning Association

1708

Journal of Strength and Conditioning Research not impair strength development in young school boys. As expected, strength training by itself does not improve aerobic capacity. Our results also suggest that training program effects even persist at the end of the DT period.

KEY WoRDS youth, strength, cardiorespiratory, schooling pupils, experimental, program recess

\section{INTRODUCTION}

$\mathrm{T}$ here is strong evidence that school-based interventions are effective in promoting physical activity levels $(4,5,8,25,28,29,31)$ by implementing welldesigned fitness programs $(26,32,33,34,36)$. Although the majority of the pediatric research has focused on activities that enhance cardiorespiratory fitness, recent findings indicate that resistance training can offer unique benefits to children and adolescents when appropriately prescribed and supervised $(2,4,13,23)$. Indeed, improvements in muscular fitness and speed and agility, rather than cardiorespiratory fitness, seem to have a positive effect on skeletal health $(13,24)$. Furthermore, children and adolescents involved in physical education classes often perform strength and endurance training concurrently in an effort to achieve specific adaptations to both forms of training $(5,12,21,29)$. On this issue, the scientific literature has produced inconclusive results. Some studies have shown that concurrent training impairs the development of strength and power but does not affect the development of aerobic condition when compared with both form of stand-alone training. Some researchers have reported that concurrent training has an inhibitory effect on the development of strength and endurance $(5,9,14)$. For example, the addition of heavy resistance training to specific team handball training skills in adolescent boys resulted in gains in maximal strength and throwing velocity, but it may have compromised gains in the production of explosive force in the leg and endurance running (10). Yet, the precise mechanisms that underlie the observed impairments in 
Journal of Strength and Conditioning Research" $\mid$ www.nsca-jscr.org

training adaptation during concurrent training have to be identified $(20,21,37)$.

Differently, in adults, concurrent training produces better strength and endurance results rather than if each strength or endurance training method is performed separately (1). In this line, physical education classes demand a balance between strength and endurance, and it seems important to training both capacities concurrently. Nevertheless, the effects of concurrent resistance and endurance training in elementary school untrained male students are yet to be investigated.

Interruptions in the training process because of illness, injury, holidays, postseason break, or other factors are normal in any kind of sport $(2,3,6)$ and also in the school context. The magnitude of this reduction may depend upon the length of the detraining (DT) period in addition to the training levels attained by the subject (20). Nevertheless, the DT period and its consequences are not well reported in sports literature and during puberty. This is important because the period of strength training cessation can produce a positive delay transformation rebound in sports-specific performance (9), which is determinant on school performance evaluation of the student.

The established hypothesis put forward in this article is that young teenage boys can significantly increase parameters of power strength (speed, jump, and throws) performances by combining physical education classes with specific training programs over a consecutive 8-week period. We also hypothesized that concurrent resistance and endurance training would have a main positive effect on power strength development of untrained school boys compared with those found when power training was applied alone. Additionally, a 12-week DT period during the summer holidays may not produce significant decreases in physical performance, in both power training group and concurrent power and endurance training group, although endurance training groups would keep some strength gains after training cessation.

Therefore, the purpose of this study was twofold: (a) to analyze the effects of power training alone and combined power and endurance training on body composition, power strength, and endurance training on a large sample of healthy school boys and (b) to assess the effects of a DT period on strength, power, and endurance performances and in body composition.

\section{Methods}

\section{Experimental Approach to the Problem}

Forty-two healthy boys recruited from a Portuguese public high school were randomly assigned to 2 experimental groups (8-week training program, twice a week, from April 12 to June $4,2010)$ and 1 control group as follows: 1 group performing power training only (GR: $n=15)$; another group performing combined power strength and endurance training (GCOM: $n=15$ ); and the third was the control group (GC: $n=12$; without a training program). All the sample subjects attended physical education classes twice a week, with a duration of
45 and 90 minutes each class, respectively. Typical physical education classes included various sports (gymnastics, team sports, athletics, dancing, and adventure sports, among others) with a clear pedagogical focus. As such, according to other researchers (30), the physical activity intensity is considered low to moderate. The participants in all the groups were asked to maintain normal eating and physical activity patterns over the duration of the study. This procedure was the same as that of Lubans et al. (18). Usually, these classes start with a jogging run for 10 minutes to general warm-up and proceed to joint mobilization and general stretches. After that, the class is divided into 2 or 3 proficiency level groups to start the main activities and sports of the class, which can be a drill or a game organized in small groups. In Portugal, a physical education class has a set of 45 minutes and another of 90 minutes twice a week.

The training program was implemented additionally to physical education classes in the same outdoor sportive facility. After a 10-minute warm-up period, both experimental groups were submitted to a power strength training program composed of 1- and 3-kg medicine balls throws performed as long and fast as possible; jumps onto a box (from 0.4 to $0.6 \mathrm{~m}$ of height), plyometric jumps $>0.4-0.6 \mathrm{~m}$ of height hurdle (only 1 foot touch on the floor among hurdles), and sets of 30- to 40-m speed running. To the GCOM group was complementarily administered a $20-\mathrm{m}$ shuttle run training exercise (15), which occurred immediately after the power strength training session. This endurance task was developed based on an individual training volume set to about $75 \%$ of the established maximum aerobic volume achieved in a previous test. After 4 weeks of training, the GCOM subjects were reassessed using $20-\mathrm{m}$ shuttle run test to readjust the volume and intensity of the $20-\mathrm{m}$ shuttle run exercise. Both GR and GCOM trained on the same day of the week (with 2/3 days between training sessions) and at the same morning hour. The subjects were encouraged to hydrate before and at the middle of a training session. All the participants were familiarized with power training drills (sprints, jumps, and ball throws) and with the 20-m shuttle run protocol. Throughout the preexperimental and experimental periods, the subjects reported their noninvolvement in additional regular exercise programs for developing or maintaining strength and endurance performance besides institutional regular physical education classes. A more detailed analysis of the program can be found in Table 1 .

Sample groups were assessed for upper and lower body explosive strength (overhead medicine ball throwing and countermovement horizontal and vertical jumps, respectively), running speed (20-m sprint run), and $\mathrm{VO}_{2}$ max (20-m shuttle run test) before and after 8 weeks of the training program. To evaluate the DT effects, all the individuals were reassessed 12 weeks after training had stopped. The DT period coincided with the summer holidays. Throughout this period, the subjects reported their noninvolvement in regular exercise programs for developing or maintaining strength 
TABLE 1. Training program design. ${ }^{*} \dagger$

\begin{tabular}{|c|c|c|c|c|c|c|}
\hline \multirow[b]{2}{*}{ Sessions } & \multicolumn{6}{|c|}{ Exercises } \\
\hline & 1 & 2 & 3 & 4 & 5 & 6 \\
\hline Chest 1 -kg medicine ball throw $\ddagger \S$ & $2 \times 8$ & $2 \times 8$ & $2 \times 8$ & $2 \times 8$ & $6 \times 8$ & $6 \times 8$ \\
\hline Chest 3-kg medicine ball throw $\ddagger \S$ & $2 \times 8$ & $2 \times 8$ & $2 \times 8$ & $2 \times 8$ & & \\
\hline Overhead $1-\mathrm{kg}$ medicine ball throw $\ddagger \S$ & $2 \times 8$ & $2 \times 8$ & $2 \times 8$ & $2 \times 8$ & $6 \times 8$ & $6 \times 8$ \\
\hline Overhead 3-kg medicine ball throw $\ddagger \widehat{S}$ & $2 \times 8$ & $2 \times 8$ & $2 \times 8$ & $2 \times 8$ & & \\
\hline $\mathrm{CMJ}$ onto a box $\$$ & $1 \times 5$ & $1 \times 5$ & $3 \times 5$ & $3 \times 5$ & $3 \times 5$ & $4 \times 5$ \\
\hline Plyometric jumps $>3$ hurdling $\$ \S$ & $5 \times 4$ & $5 \times 4$ & $5 \times 4$ & $5 \times 4$ & $2 \times 3$ & $2 \times 3$ \\
\hline Sprint running $(\mathrm{m}) \ddagger \S$ & $4 \times 20$ & $4 \times 20$ & $3 \times 20$ & $3 \times 20$ & $3 \times 20$ & $3 \times 20$ \\
\hline \multirow[t]{2}{*}{ 20-m Shuttle run (MAV) (\%)§ } & 75 & 75 & 75 & 75 & 75 & 75 \\
\hline & 7 & 8 & 9 & 10 & 11 & 12 \\
\hline \multicolumn{7}{|l|}{ Chest 1 -kg medicine ball throw $\neq \S$} \\
\hline \multicolumn{7}{|l|}{ Overhead $1-\mathrm{kg}$ medicine ball throw $\ddagger \S$} \\
\hline Overhead 3-kg medicine ball throw $\ddagger \S$ & $2 \times 8$ & $2 \times 8$ & $3 \times 8$ & $3 \times 8$ & $3 \times 8$ & \\
\hline $\mathrm{CMJ}$ onto a box $\ddagger \S$ & $4 \times 5$ & $5 \times 5$ & $5 \times 5$ & $5 \times 5$ & $5 \times 5$ & $4 \times 5$ \\
\hline Plyometric jumps $>3$ hurdling $\$ \S$ & $3 \times 3$ & $4 \times 3$ & $4 \times 3$ & $4 \times 3$ & $4 \times 3$ & \\
\hline Sprint running $(\mathrm{m}) \ddagger \S$ & $4 \times 30$ & $4 \times 30$ & $4 \times 30$ & $4 \times 30$ & $4 \times 30$ & $3 \times 40$ \\
\hline \multirow[t]{2}{*}{ 20-m Shuttle run (MAV)§ } & $75 \%$ & Test M & $75 \%$ & $75 \%$ & $75 \%$ & $75 \%$ \\
\hline & 13 & 14 & 15 & 16 & & \\
\hline \multicolumn{7}{|l|}{ Chest 1 -kg medicine ball throw $\ddagger \S$} \\
\hline Chest 3-kg medicine ball throw $\ddagger \S$ & $2 \times 5$ & $1 \times 5$ & & & & \\
\hline Overhead $1-\mathrm{kg}$ medicine ball throw $\ddagger \S$ & & $3 \times 8$ & $2 \times 8$ & $2 \times 8$ & & \\
\hline Overhead 3-kg medicine ball throw $\ddagger \widehat{\S}$ & $3 \times 8$ & & & & & \\
\hline CMJ onto a box $\ddagger \S$ & $4 \times 5$ & $2 \times 5$ & $2 \times 4$ & $2 \times 4$ & & \\
\hline Plyometric jumps $>3$ hurdling $\$ \S$ & $4 \times 3$ & $3 \times 3$ & & & & \\
\hline Sprint running $(\mathrm{m}) \ddagger \S$ & $3 \times 40$ & $4 \times 40$ & $2 \times 30$ & $2 \times 30$ & & \\
\hline 20-m Shuttle run (MAV) (\%)§ & 75 & 75 & 75 & 75 & & \\
\hline
\end{tabular}

${ }^{\star} \mathrm{CMJ}=$ countermovement jump; MAV = maximum individual aerobic volume; GCOM = concurrent resistance and endurance training; $\mathrm{GR}=$ power strength training protocol.

$\dagger$ Medicine ball throwing and jump onto a box the first number corresponds to sets and the second corresponds to repetitions. For sprint running, the first number corresponds to sets and the second corresponds to the distance to run. For the 20-m shuttle run training, each girl ran each session (until test M) $75 \%$ of the maximum individual aerobic volume performed on the pretest and after this test $\mathrm{M}$ moment until the program end, ran $75 \%$ of the maximum individual aerobic volume performed on test $\mathrm{M}$.

$\ddagger G R$.

§GCOM.

and endurance performance. The testing assessment procedures were always conducted in the same indoor environment, at the same daily and weekly schedule (from March to September 2010). Each subject was familiarized with the power training tests (sprints, jumps, and ball throws) and with the $20-\mathrm{m}$ shuttle run test. All data collection was performed by the same investigator and after a general warmup of 10 minutes.

\section{Subjects}

A sample of 42 healthy boys recruited from a Portuguese public high school (from seventh and ninth grades) was used in this study. To fulfill the ethical procedures of the Helsinki statement, an informed consent was obtained before all testing adolescents' parents. Efforts were made to recruit subjects for making comparable groups. Maturity level based on Tanner stages (5) was self-assessed. There were no significant differences $(p>0.05)$ between groups for age or Tanner ratings, either in anthropometrics or performance variables at the beginning of the protocol. No subject had regularly participated in any form of strength training before this experiment. The following exclusion criteria were used: subjects with a chronic pediatric disease or with an orthopedic limitation.

\section{Testing Procedures}

Anthropometric Assessment. Total height (meters) was assessed according to international standards for anthropometric assessment (19), with a Seca 264 Stadiometer (Hamburg, Germany). Body composition variables were assessed using a Tanita body composition analyzer; model TBF-300 (Tanita Corporation of America, Inc., Arlington Heights, IL, USA) with a ratio of $1-75 \%$. These parameters were assessed before 
Journal of Strength and Conditioning Research" | www.nsca-jscr.org

TABLE 2. Descriptive (mean $\pm S D$ ) characteristics of the participants during 3 testing trials $(M 1, M 2$, and $M 3)$ for all the groups.*

\begin{tabular}{|c|c|c|c|c|c|c|}
\hline \multirow[b]{2}{*}{ Variable } & \multirow[b]{2}{*}{ Group } & \multicolumn{3}{|c|}{$x \pm \sigma$} & \multicolumn{2}{|c|}{$p$ Value } \\
\hline & & M1 & M3 & M3 & (M1-M2) & (M2-M3) \\
\hline \multirow[t]{3}{*}{ Body weight (kg) } & $\mathrm{GC}$ & $56.5 \pm 11.2$ & $56.9 \pm 11.0$ & $56.8 \pm 4.9$ & 0.22 & 0.14 \\
\hline & GR & $59.2 \pm 16.2$ & $58.3 \pm 16.0$ & $58.9 \pm 16.7$ & 0.11 & 0.28 \\
\hline & GCOM & $51.4 \pm 8.2$ & $51.3 \pm 8.2$ & $50.8 \pm 7.3$ & 0.85 & 0.03 \\
\hline \multirow[t]{3}{*}{ Total standing height $(\mathrm{cm})$} & GC & $163.8 \pm 9.9$ & $164.5 \pm 9.8$ & $167.1 \pm 9.7$ & 0.06 & 0.08 \\
\hline & GR & $161.8 \pm 12.2$ & $163.6 \pm 11.5$ & $163.9 \pm 11.8$ & 0.00 & 0.00 \\
\hline & GCOM & $159.5 \pm 8.1$ & $160.2 \pm 8.0$ & $161.3 \pm 7.9$ & 0.09 & 0.01 \\
\hline \multirow[t]{3}{*}{$\mathrm{BMI}\left(\mathrm{kg} \cdot \mathrm{m}^{-2}\right)$} & $\mathrm{GC}$ & $21.0 \pm 3.4$ & $21.0 \pm 3.6$ & $20.6 \pm 3.0$ & 0.74 & 0.17 \\
\hline & GR & $22.3 \pm 4.6$ & $21.6 \pm 4.7$ & $22.0 \pm 5.0$ & 0.00 & 0.26 \\
\hline & GCOM & $20.1 \pm 2.1$ & $19.9 \pm 2.3$ & $20.3 \pm 2.7$ & 0.22 & 0.22 \\
\hline \multirow[t]{3}{*}{ Body fat (\%) } & GC & $15.0 \pm 6.4$ & $14.1 \pm 7.1$ & $12.8 \pm 6.6$ & 0.11 & 0.74 \\
\hline & GR & $18.2 \pm 9.2$ & $15.8 \pm 8.4$ & $17.0 \pm 8.6$ & 0.00 & 0.36 \\
\hline & GCOM & $14.5 \pm 4.6$ & $12.7 \pm 4.6$ & $12.5 \pm 4.6$ & 0.00 & 0.75 \\
\hline
\end{tabular}

${ }^{*} x=$ mean; $\sigma=S D ; \mathrm{M} 1=$ before training program; $\mathrm{M} 2=$ after training program; $\mathrm{M} 3=$ after the detraining; period; $p(\mathrm{M} 1-\mathrm{M} 2)=p$ value for comparison between the second and first moments; $p(\mathrm{M} 2-\mathrm{M} 3)=p$ value for the comparison between the third and second moments; $\mathrm{GC}=$ control group; $\mathrm{GR}=$ resistance training group; GCOM = concurrent resistance and endurance training.

any physical performance test. The subjects were measured when they were wearing shorts and t-shirts (shoes and socks were asked to be removed).

Overhead Medicine Ball Throwing. An overhead medicine ball throw test was used to evaluate the upper body ability to generate muscular actions at a high rate of speed. Before baseline tests, each subject underwent one familiarization session and was counseled on proper overhead throwing with different weighted balls. Pretests, posttests, and DT measurements were conducted on maximal throwing velocity using medicine balls (Bhalla International, Vinex Sports, Meerut, India) weighing $1 \mathrm{~kg}$ (Vinex, model VMB-001R, perimeter $0.72 \mathrm{~m}$ ) and $3 \mathrm{~kg}$ (Vinex, model VMB-003R, perimeter $0.78 \mathrm{~m}$ ). A general warm-up period of 10 minutes, which included throwing the $1-$ and $3-\mathrm{kg}$ weighted balls, was allowed. While standing, the subjects held medicine balls with 1 and $3 \mathrm{~kg}$ in both hands in front of the body with arms extended. The students were instructed to throw the ball over their heads as far and fast as possible. A countermovement was allowed during the action. One minute of rest among 5 trials was given. Only the best throw was considered for analysis. The ball throwing distance was recorded to the closest centimeter as proposed by van Den Tillaar and Marques (35). This was possible because polyvinyl chloride medicine balls were used and when these fall on the copolymer polypropylene floor, a visible mark was made. The ICC of data for 1- and 3-kg medicine ball throwing was 0.97 and 0.99 , respectively.
Countermovement Vertical Jump. To monitor the effectiveness of an athlete's conditioning program, the standing vertical jump test of leg power was used. The vertical jump test was conducted on a contact mat connected to an electronic power timer, control box, and handset (Globus Ergojump, Codogné, Italy). From a standing position, with the feet shoulder width apart and the hands placed on the pelvic girth, the boys performed a countermovement with the legs before jumping. Such movement makes use of the stretch-shorten cycle, in which the muscles are prestretched before shortening in the desired direction (17). They were informed that they should try to jump vertically as high as possible. Each participant performed 3 jumps with a 1-minute recovery between attempts. The highest jump (centimeters) was recorded. The countermovement vertical jump (CMVJ) showed an ICC of 0.95

Countermovement Standing Long Jump. In a standing long jump, the jumper aimed to project his body for a maximum horizontal distance beyond the take-off line. The jumper started from a static standing position with feet shoulder width apart and then generated a large take-off speed by using a countermovement coupled with the hands placed on the pelvic girth and a double-leg take-off. The take-off is characterized by a large forward lean of the body, and during the flight phase, the jumper swings the legs forward underneath the body in preparation for landing. The jumper landed with a prominent forward lean of the trunk and with the feet extended well ahead of the hips. To be credited with a successful jump, the jumper must retain balance 
TABLE 3. Mean $\pm S D$ of CMVJ, CMSLJ, 1 - and 3-kg medicine ball throwing, running speed, and $\dot{\mathrm{V}}_{2}$ max in all the 3 testing trials (M1, M2, and M3) for each group.*

\begin{tabular}{|c|c|c|c|c|c|c|}
\hline & \multirow[b]{2}{*}{ Group } & \multicolumn{3}{|c|}{$x \pm \sigma$} & \multicolumn{2}{|c|}{$p$ Value } \\
\hline & & M1 & M2 & M3 & $(\mathrm{M} 1-\mathrm{M} 2)$ & (M2-M3) \\
\hline \multirow[t]{3}{*}{ CM vertical jump (cm) } & GC & $0.288 \pm 0.07$ & $0.317 \pm 0.07$ & $0.317 \pm 0.09$ & 0.15 & 0.71 \\
\hline & GR & $0.293 \pm 0.07$ & $0.306 \pm 0.07$ & $0.277 \pm 0.08$ & $0.04 \dagger$ & 0.14 \\
\hline & GCOM & $0.298 \pm 0.08$ & $0.316 \pm 0.09$ & $0.295 \pm 0.10$ & $0.02 \dagger$ & 0.37 \\
\hline \multirow{3}{*}{$\mathrm{CM}$ standing long jump (m) } & $\mathrm{GC}$ & $1.70 \pm 0.37$ & $1.63 \pm 0.33$ & $1.62 \pm 0.51$ & 0.26 & 0.72 \\
\hline & GR & $1.49 \pm 0.27$ & $1.56 \pm 0.30$ & $1.47 \pm 0.36$ & $0.00 \dagger$ & 0.17 \\
\hline & GCOM & $1.67 \pm 0.31$ & $1.74 \pm 0.32$ & $1.54 \pm 0.43$ & $0.00 \dagger$ & 0.12 \\
\hline \multirow[t]{3}{*}{ 1-kg Medicine ball throwing $(\mathrm{m})$} & GC & $8.23 \pm 1.47$ & $8.31 \pm 1.71$ & $8.89 \pm 1.75$ & 0.08 & 0.11 \\
\hline & GR & $7.50 \pm 1.70$ & $8.15 \pm 1.62$ & $8.13 \pm 1.45$ & $0.00 \dagger$ & 0.31 \\
\hline & GCOM & $7.26 \pm 1.60$ & $7.59 \pm 1.73$ & $7.71 \pm 2.27$ & $0.04 \dagger$ & 0.37 \\
\hline \multirow{3}{*}{ 3-kg Medicine ball throwing $(\mathrm{m})$} & GC & $5.02 \pm 0.91$ & $5.01 \pm 1.19$ & $5.35 \pm 1.30$ & 0.10 & 0.15 \\
\hline & GR & $4.66 \pm 0.98$ & $5.12 \pm 1.08$ & $5.10 \pm 0.99$ & $0.00 \dagger$ & 0.29 \\
\hline & GCOM & $4.60 \pm 1.12$ & $5.11 \pm 1.17$ & $5.03 \pm 1.25$ & $0.04 \dagger$ & 0.97 \\
\hline \multirow{3}{*}{ Running speed 20 m (s) } & $\mathrm{GC}$ & $4.13 \pm 0.55$ & $4.12 \pm 0.48$ & $3.52 \pm 0.49$ & 0.95 & 0.12 \\
\hline & GR & $4.54 \pm 0.49$ & $4.05 \pm 0.42 \ddagger$ & $4.04 \pm 0.36$ & $0.00 \dagger$ & 0.43 \\
\hline & GCOM & $4.38 \pm 0.59$ & $3.81 \pm 0.28$ & $3.83 \pm 0.50$ & $0.00 \dagger$ & 0.93 \\
\hline \multirow[t]{3}{*}{$\dot{\mathrm{V}} \mathrm{O}_{2} \max \left(\mathrm{ml} \cdot \mathrm{kg}^{-1} \cdot \mathrm{min}^{-1}\right)$} & $\mathrm{GC}$ & $48.5 \pm 5.3$ & $47.4 \pm 5.5$ & $44.4 \pm 8.1$ & 0.67 & 0.52 \\
\hline & GR & $45.2 \pm 6.4$ & $46.8 \pm 6.5$ & $42.1 \pm 5.2$ & 0.10 & $0.04 \dagger$ \\
\hline & GCOM & $49.1 \pm 6.7$ & $51.2 \pm 6.7$ & $51.7 \pm 6.6$ & $0.01 \dagger$ & 0.83 \\
\hline
\end{tabular}

${ }^{*} x=$ mean; $\sigma=S D ; C M=$ countermovement; $M 1=$ before training program; $\mathrm{M} 2=$ after training program; $\mathrm{M} 3=$ after the detraining period; $p(\mathrm{M} 1-\mathrm{M} 2)=p$ value for comparison between the second and first moments; $p(\mathrm{M} 2-\mathrm{M} 3)=p$ value for comparison between third and second moments; GC = control group; GR = resistance training group; GCOM = concurrent resistance and endurance training; $\mathrm{CMVJ}=$ countermovement vertical jump.

$\ddagger$ Significant changes between moments.

after landing and not fall backward into the pit. A standing long jump performance was quantified by the total jump distance, which is the distance from the take-off line to the nearest break in the landing area made by the heels at landing (38). A fiberglass tape measure (Vinex, MST-50M) was extended across the floor and used to measure horizontal distance. Each participant completed 3 trials with a 1-minute recovery between trials using a standardized jumping protocol to reduce interindividual variability. The greatest distance (centimeters) of the 2 jumps was taken as the test score. The CM standing long jump (CMSLJ) showed an ICC of 0.96 .

Sprint Running. This test was performed in an indoor school physical education facility with a copolymer polypropylene floor; the subjects wore adapted indoor shoes. The time to run $20 \mathrm{~m}$ was obtained using photocells (Brower Timing System, Fairlee, VT, USA). The time to run the distance was recorded using a digital and automatic chronometer commanded by the cell pad and a pair of photocells positioned above the 20 -m line. At the start moment, each subject trod the cell pad using their right hand with the time being recorded from when the subjects intercepted the photocell beam. All the subjects were encouraged to run as fast as possible and to decelerate only after listening to the beep emitted by the last photocells pair. Each student repeated the same procedure for 3 attempts, and only the best time taken to cover the $20-\mathrm{m}$ distance in the sprint test was used in data analysis. A rest period of 10 minutes among attempts was accomplished. The sprint running (time) showed an ICC of 0.97 .

Twenty-Meters Shuttle Run. This test involves continuous running between 2 lines ( $20 \mathrm{~m}$ apart in time) to recorded beeps. The time between recorded beeps decreased each minute (level). We used the common version with an initial running velocity of $8.5 \mathrm{~km} \cdot \mathrm{h}^{-1}$ and increments of $0.5 \mathrm{~km} \cdot \mathrm{h}^{-1}$ each minute (15). Estimated $\dot{\mathrm{V}}_{2} \max$ (milliliters per kilogram per minute) was calculated by using Léger's equation (15), which is based on the level and number of shuttles reached before the boys were unable to keep up with the audio recording. The $20-\mathrm{m}$ shuttle run test showed an ICC of 0.96 .

\section{Statistical Analyses}

Standard statistical methods were used for the calculation of the means and $S D s(x \pm \sigma)$. One-way analysis of variance (ANOVA) was used to determine any differences among the 3 groups' initial power strength, running speed, endurance, and anthropometry. The training related effects 
Journal of Strength and Conditioning Research" $\mid$ www.nsca-jscr.org

were assessed using a 2-way ANOVA with repeated measures (groups $\times$ moment). Selected absolute changes in each moment were analyzed via the -way ANOVA. The $p \leq 0.05$ criterion was used for establishing statistical significance.

\section{Results}

There were no significant differences $(p>0.05)$ between groups for age or Tanner stages, in either anthropometrics or performance variables at the beginning of the protocol $(p>0.05)$. Body fat (BF) decreased significantly $(p=0.00)$ from the pretraining to the posttraining period in both the GR and GCOM groups (Table 2); however, no significant differences were found between groups. No significant changes were observed for the total standing height, body weight, and body mass index (BMI) in both GC and GCOM groups. The GR significantly changed in height $(+1.2 \%$, $p=0.004)$, BMI $(-0.4 \%, p=0.00)$, and $\mathrm{BF}(-10 \%, p=0.00)$, whereas GCOM only decreased in BF but was not significantly different from GR. From the pretraining to the posttraining period, no differences were observed between experimental groups for performance variables, that is, the subjects from the GCOM group did not take advantage of the subjects from the GR group in jumps, running speed, and balls throw tests. However, $\dot{\mathrm{VO}}_{2} \mathrm{max}$ increased significantly in the GCOM $(+4.6 \%, p<0.01)$, but it remained unchanged in both the GC and GR groups. The magnitude of changes in the 1- and 3-kg ball throw distance, height in CMVJ, length in CMSLJ, and time to run $20 \mathrm{~m}$ was similar in both the GR and GCOM groups (Table 3).

The DT period resulted in decreased body weight $(-2.7 \%$, $p=0.03$ ) for GCOM (Table 2), whereas it remained constant for the GR and GC groups. Body height increased significantly for GR $(+0.6 \%, p=0.00)$ and GCOM $(+0.7 \%, p=0.01)$. No significant changes were observed in the BMI from the posttraining to the DT period in any group. No significant changes were observed in $\mathrm{BF}$ loss in any of the experimental groups. No significant changes were observed in 1- and 3-kg medicine ball throw gains after the DT period in any groups (Table 3). No significant changes in the vertical jump height, horizontal jump length, and time to run $20 \mathrm{~m}$ after the DT period were observed after the DT period (Table 3). The estimated $\dot{\mathrm{V}}_{2}$ max, however, decreased after the DT period in the GR group $(-6.8 \%, p=0.04)$ but not in the GCOM. When comparing groups, significant differences were not found.

\section{Discussion}

To the best of our knowledge, no other study has established the effect of an 8-week school-based endurance and resistance training program and DT on strength, power, and body composition in adolescent boys, performed additionally to the physical education lessons. Thus, it is difficult to compare the present results with the results of other studies that have investigated physical training cessation, because they differ markedly in a number of factors, including the sample and the method of measurement. The primary findings of this study indicate that both concurrent resistance and endurance training and resistance training alone may be a positive training stimulus to enhance explosive strength and aerobic condition in healthy schooled boys. Our findings are in agreement with those of the Gorostiaga et al. (10) and Chtara et al. (1) studies conducted with adults. Simultaneously, our results contradict studies, which reported an impairment of concurrent training on performance variable development (29). Additionally, both training regimens also showed a positive effect on $\mathrm{BF}$ loss in adolescent school boys. Therefore, the present results may suggest that concurrent resistance and endurance training seems to be an effective, well-rounded exercise program that can be prescribed as a means to improve the initial or general strength in healthy school boys. Moreover, both training program regimen effects persisted as long as upper and lower limb strength gains were maintained during 12 weeks of the DT period. Concordantly, the group submitted to the strength and endurance program did not show estimated $\mathrm{V}_{2}$ max loss in the DT period.

The magnitude of decrease observed in the $\mathrm{BF}$ was not significantly different between the GR and GCOM groups. We did not find any change in body weight for any group. It should be highlighted that body weight does not always explain the true body composition, and therefore, despite the fact that we did not find any body weight changes, we found significant losses in the $\mathrm{BF}$ in both experimental groups. However, we did not find significant differences between experimental groups. These results may suggest that there is no major positive effect of concurrent resistance and endurance training when the BF loss occurs. Furthermore, the current results are in agreement with those of the research conducted by Watts et al. (39) that examined an independent influence of 8 weeks of combined resistance and aerobic training in 19 obese adolescents aged 12- to 16-years. In this, although bodyweight and BMI did not change with exercise, significant improvements in central adiposity were observed after the 8-week circuit-training program (39).

A significant increase was observed for upper limb explosive strength (e.g., medicine ball throw with 1 and $3 \mathrm{~kg}$ ), in both the GCOM and GR groups. These data may suggest a main positive effect of resistance training on explosive strength ability independently of the type of treatment performed. In accordance with the upper body strength results, the explosive power of lower limbs revealed by the CMVJ and CMSLJ performance also increased significantly for both experimental groups. Few studies, however, have compared the effects of different methods of organizing training workouts. Here, for example, Sale et al. (27) could observe that concurrent resistance and endurance training applied on separate days produced superior gains to those produced by concurrent training on the same day. Although the training programs were conducted otherwise constantly, alternate-day training was more efficient in 
producing maximal leg press strength gains than was sameday training. This suggests that the interference effect may also be true when the overall frequency and volume of training are higher than that in this particular study. Also, Ingle et al. (11) using a combination of resistance training and plyometric program found that the experimental group experienced a small improvement in performance over the training intervention period. Our results also demonstrated that the endurance training does not positively affect strength development in school boys. In addition, however, this research showed that concurrent resistance and endurance training do not impair strength development. Unfortunately, it is difficult to compare results in the scientific literature when studies differ markedly in their design factors including load characteristics, context, equipment, scheduling of training sessions, and training history of subjects $(16,37)$. Therefore, further research is required to investigate these causes and identify other possible mechanisms responsible for the observed inhibition in strength development after concurrent training (27).

Running speed increased significantly in all experimental groups. In agreement with the findings of previous studies (20), these results seem to indicate that additional endurance training does not have an additional effect over strength training in enhancing running speed in young boys. On the other hand, all the students approached various sports during Physical Education classes. Although physical activity intensity can be considered low to moderate, some sports (for instance, soccer and basketball) elicit high-intensity performances (sprints) and low-intensity periods, which could have enhanced running speed performance.

An inhibition in strength or endurance adaptation as a consequence of concurrent training has been reported (37). Nevertheless, this study could observe a significant enhancement in $\dot{V}_{\mathrm{O}_{2}} \max$ (milliliters per kilogram per minute) only for GCOM, suggesting that the resistance training program component was not effective to an increase in aerobic fitness for young school boys. Our data suggest that dependent variable selection can influence the conclusions made with respect to changes in strength and endurance as a result of concurrent training. However, the differences in the design of concurrent training interventions, such as the mode, duration, and intensity of training, may influence whether any interference in strength or endurance development is observed. Clearly, the interaction between strength and endurance training is a complex issue, and it may still be possible to design specific concurrent training regimens that can minimize or possibly avoid any interference effects.

Twelve consecutive weeks during the summer holidays were taken as the detrained period. All the sample subjects had no formal physical activity (Physical Education lessons or institutional training programs) during the DT period. Only the GCOM significantly decreased in body weight $(-1.7 \%$, $p=0.03)$. The Total Standing Height variable for both the experimental groups significantly increased from posttraining to DT. There was no significant difference in the BMI on the GR group from posttraining to DT. Additionally, there was no significant difference in the $\mathrm{BF}$ percentage loss between GR and GCOM during the intervention period. Thus, we can assume that the sustainment of $\mathrm{BF}$ obtained with training program participation is evident for several weeks after the program had been completed. Conversely to the posttraining moment, all the groups had shown no significant loss performance in CMVJ and CMSLJ. In speed running, a significant loss performance was expected, but this was not found in both GR and GCOM. A possible loss was expected because speed running is strongly affected by nervous system adaptation and phosphocreatine reserves; however, this was not observed (7). In the 1- and 3-kg medicine ball throw distance test, no significant changes were observed for the experimental groups, which implied a sustained effect of training in this explosive task. Our results are in disagreement with Ingle et al.'s findings (11), which showed that in a 12-week DT period, the experimental group experienced reductions for all the resistance exercises that ranged from -16.3 to $-30.3 \%$. The control group had also no differences in performance marks for both the 1- and 3-kg medicine ball throw distance tests. Therefore, it must be suggested that explosive strength gains induced by both the training programs were maintained after a DT period of 12 weeks, because strength is determined, among other factors, by muscular mass. Faigenbaum et al. (6) showed that 8 weeks of DT led to significant losses of leg extension $(-28.1 \%)$ and chest press $(-19.3 \%)$ strength, whereas control group strength scores remained relatively unremarkable.

Finally, the $\dot{\mathrm{V}}_{2} \max$ (milliliters per kilogram per minute) remained stable for the GCOM, except for the GR where a significantly loss $(-6.8 \%)$ was observed. Another study (22) found that changes are more moderate in recently trained subjects (compared with that in highly trained subjects) in the short term, but recently acquired $\dot{\mathrm{VO}}_{2}$ max gains are completely lost after training stoppage $>4$ weeks. Conversely, our results show that the GCOM maintained the $\dot{\mathrm{V}}_{2}$ max gains even after 12 weeks of DT. The DT effect over $\dot{\mathrm{V}}_{2}$ max has been poorly studied in nonadult and nonsportive samples. Hence, because of the small sample size and the lack of a prestudy power analysis to determine adequate effect size for this study, we suggest that our subgroup analyses and results must be interpreted with caution.

Our results suggest that a concurrent resistance and endurance school-based training program seems considerably effective in both strength and endurance fitness features of school-aged boys. However, the resistance training program also produced identical results in strength development. In brief, this study indicates that concurrent training is an effective, well-rounded exercise program that can be performed to improve initial or general strength in healthy school boys. Our results also suggest that training program effects persist even at the end of the DT period. These effects include body composition effects, and physical fitness components such as 
Journal of Strength and Conditioning Research $\mid$ www.nsca-jscr.org

strength and endurance. Future research studies should examine the interference effects arising from the order of resistance and endurance training exercise program in strength enhancement.

\section{Practical Applications}

Simultaneously performing resistance and endurance training in the same workout not only does not impair strength development in healthy school boys but it also seems to be an effective, well-rounded exercise program that can be prescribed as a means to improve initial or general strength. This should be considered in the designing of strength training school-based programs to improve its efficiency. Furthermore, school-based programs should be implemented because training program effects persist at the end of summer holidays on body composition and physical fitness level.

\section{Acknowledgments}

The authors thank the children and their parents for participating in this study and gratefully acknowledge Manuela Costeira (the School Principal of EB Poceirão) and her management team for allowing the use of the training equipment in this study and school facilities, Jorge Romão (the School Principal of EB2,3 Pegões) and his management team for allowing the use of the school facilities. The authors would also like to graciously thank the reviewers who took the time to critique this manuscript. The authors have no professional relationships with any companies or manufacturers identified in this study. The results of this study do not constitute endorsement of the product either by the authors or by the National Strength and Conditioning Association.

\section{REFERENCES}

1. Chtara, MK, Chamari, M, Chaouachi, A, Chaouachi, D, Koubaa, Y, Feki, GP, Millet, and Amri, M. Effects of intra-session concurrent endurance and strength training sequence on aerobic performance and capacity. Br J Sports Med 39: 555-560, 2005.

2. Faigenbaum, AD, Kraemer, WJ, Blimkie, CJ, Jeffreys, I, Micheli, LJ, Nitka, M, and Rowland, TW. Youth resistance training: Updated position statement paper from the national strength and conditioning association. J Strength Cond Res 23(Suppl. 5): S60-S79, 2009.

3. Faigenbaum, $\mathrm{AD}$ and Mediate, P. Effects of medicine ball training on fitness performance of high-school physical education students. Phys Educ 63: 160-167, 2006.

4. Faigenbaum, $\mathrm{AD}$ and Myer, GD. Pediatric resistance training: Benefits, concerns, and program design considerations. Curr Sports Med Rep 9: 161-168, 2010

5. Faigenbaum, AD, Westcott, WL, Loud, RL, and Long, C. The effects of different resistance training protocols on muscular strength and endurance development in children. Pediatrics 104: 1-7, 1999.

6. Faigenbaum, AD, Westcott, WL, Micheli, LJ, Outerbridge, AR, Long, CJ, Larosa-Loud, R, and Zaichkowskt, LD. The effects of strength training and detraining on children. J Strength Cond Res 10: 109-114, 1996

7. Folland, JP and Williams, AG. The adaptations to strength training morphological and neurological contributions to increased strength. Sports Med 37: 145-168, 2007.
8. Fox, KR and Riddoch, C. Charting the physical activity patterns of contemporary children and adolescents. Proc Nutr Soc 59: 497-504, 2000.

9. Garrido, N, Marinho, DA, Reis, VM, Van Den Tillaar, R, Costa, AM, Silva, AJ, and Marques, MC. Does concurrent dry land strength and aerobic training inhibit strength and swimming performance in young competitive swimmers? J Sports Sci Med 9: 300-310, 2010.

10. Gorostiaga, EM, Izquierdo, M, Iturralde, P, Ruesta, M, and Ibáñez, J. Effects of heavy resistance training on maximal and explosive force production, endurance and serum hormones in adolescent handball players. Eur J Appl Physiol 80: 485-493, 1999.

11. Ingle, L, Sleap, M, and Tilfrey, K. The effect of a complex training and detraining programme on selected strength and power variables in early pubertal boys. J Sports Sci 24: 987-997, 2006.

12. Izquierdo-Gabarren, M, Exposito, RJ, Garcia-Pallare, J, Medina, L, Villareal, E, and Izquierdo, M. Concurrent endurance and strength training not to failure optimizes performance gains. Sci Sports Exerc 42: 1191-1199, 2010

13. Kemper, HCG, Twisk, JWR, Van Mechelen, W, Post, GB, Roos, JC, and Lips, P. Fifteen-year longitudinal study in young adults on the relation of physical activity and fitness with the development of the bone mass: The Amsterdam growth and health longitudinal. Study Bone 27: 847-853, 2000.

14. Kraemer, WJ, Patton, JF, Gordon, SE, Harman, EA, Deschenes, MR, Reynolds, K, Newton, RU, Triplett, NT, and Dziados, JE. Compatibility of high-intensity strength and endurance training on hormonal and skeletal muscle adaptations. JAppl Physiol 78: 976989, 1995

15. Léger, LA, Mercier, D, Gadoury, C, and Lambert, J. The multistage 20-meter shuttle run test for aerobic fitness. J Sports Sci 6: 93-101, 1988 .

16. Leveritt, M, Abernethy, PJ, Barry, BK, and Logan, PA. Concurrent strength and endurance training: A review. Sports Med 28: 413-427, 1999.

17. Linthorne, NP. Analysis of standing vertical jumps using a force platform. Am J Phys 11: 1198-1204, 2001.

18. Lubans, DR, Sheaman, C, and Callister, R. Exercise adherence and intervention effects of two school-based resistance training programs for adolescents. Prev Med 50: 56-62, 2010.

19. Marfell-Jones, M, Olds, T, Stewart, A, and Carter, L. International Standards for Anthropometric Assessment. Potchefstroom, South Africa: ISAK, 2006

20. Marques, MC, Van De Tillaar, R, Vescovi, JD, and González-Badillo, JJ. Changes in strength and power performance in elite senior female professional volleyball players during the in-season: A case study. $J$ Strength Cond Res 22: 1147-1155, 2008.

21. Michael, L, Abernethy, PJ, Barry, BK, and Logan, PA. Concurrent strength and endurance training: A review. Sports Med 28: 413-427, 1999.

22. Mujika, I and Padilla, S.Cardiorespiratory and metabolic characteristics of detraining in humans. Med Sci Sports Exerc3: 413-421, 2001.

23. Myer, GD and Wall, EJ. Resistance training in the young athlete. Oper Tech Sports Med 14: 218-230, 2006.

24. Ortega, FB, Ruiz, JR, Castillo, MJ, and Sjöström, M. Physical fitness in childhood and adolescence: A powerful marker of health fitness as a health marker in young people. Int J Obes 32: 1-11, 2008.

25. Ribeiro, I, Parra, D, Torres, A, Pratt, M, Legetic, B, Malta, D, Ramos, LR, Simões, E, and Brownson, RC. School-based physical education programs: Evidence-based physical activity interventions for youth in Latin America. Global Health Prom 17: $5-15,2010$

26. Ridgers, ND, Stratton, G, Fairclough, SJ, and Twisk, JW. Children's physical activity levels during school recess: A quasi-experimental intervention study. Int J Behav Nutr Phys Act 4: 19, 2007. 
27. Sale, DG, McDougall, JD, Jacobs, I, and Garner, S. Interaction between concurrent strength and endurance training. J Appl Physiol 68: 260-270, 1990 .

28. Sallis, J, McKenzie, TL, Alcaraz, JE, Kolody, B, Faucette, N, and Hovell, ME. The effects of a 2 -year physical education program (SPARK) on physical activity and fitness in elementary school students. Am J Public Health 87: 1328-1334, 1997.

29. Shaw, BS, Shaw, I, and Brown, GA. Comparison of resistance and concurrent resistance and endurance training regimes in the development of strength. J Strength Cond Res 23: 2507-2514, 2009.

30. Simons-Morton, BG, Taylor, WC, Snider, SA, Huang, IW, and Fulton, JE. Observed levels of elementary and middle school children's physical activity during physical education classes. Prev Med 23: 437-441, 1994.

31. Simons-Morton, BG, Taylor, WC, Snider, SA, and Huang, IW. The physical activity of fifth grade students during physical education. Am J Public Health 83: 262-265, 1993.

32. Sluijs, EM, McMinn, AM, and Griffin, SJ. Effectiveness of interventions to promote physical activity in children and adolescents: Systematic review of controlled trials. Br J Sports Med 42: 653-657, 2007.

33. Strong, WB, Malina, RM, Blimkie, CJ, Daniels, SR, Dishman, RK, Gutin, B, Hergenroeder, AC, Must, A, Nixon, PA, Pivarnik, JM,
Rowland, T, Trost, S, and Trudeau, F. Evidence based physical activity for school-age youth. J Pediatr 146: 732-737, 2005.

34. Sweeting, HN. Measurement and definitions of obesity in childhood and adolescence: A field guide for the uninitiated. Nutr $J$ 6: 32, 2007.

35. van den Tillaar, R and Marques, MC. Effect of two different throwing training programs with same workload on throwing performance with soccer ball. Int J Sports Phys Perf 4: 747-484, 2009.

36. van Sluijs, E, McMinn, AM, and Griffin, SJ. Effectiveness of interventions to promote physical activity in children and adolescents: Systematic review of controlled trials. BMJ 335: 703, 2007.

37. Villarreal, ES, Kellis, E, Kraemer, WJ, and Izquierdo, M. Determining variables of plyometric training for improving vertical jump height performance: A meta-analysis. J Strength Cond Res 2 : 495-506, 2009

38. Wakai, M and Linthorne, NP. Optimum take-off angle in the standing long jump. Human Mov Sci 24: 81-96, 2005.

39. Watts, K, Beye, P, Siafarikas, A, Davis, EA, Jones, TW, O'driscoll, G, and Green, DJ. Exercise training normalises vascular dysfunction and improves central adiposity in obese adolescents. $\mathrm{J} \mathrm{Am} \mathrm{Coll}$ Cardiol 43: 1823-1827, 2004. 